\title{
INTERSECTION THEOREMS FOR SYSTEMS OF SETS (III)
}

\author{
Dedicated to the memory of Hanna Neumann \\ P. ERDÖS, E. C. MILNER and R. RADO
}

(Received 17 November 1972)

Communicated by M. F. Newman

\section{Introduction}

A system or family $\left(A_{\gamma}: \gamma \in N\right)$ of sets $A_{\gamma}$, indexed by the elements of a set $N$, is called an $(a, b)$-system if $|N|=a$ and $\left|A_{\gamma}\right|=b$ for $\gamma \in N$. Expressions such as " $(a,<b)$-system" are self-explanatory. The system $\left(A_{\gamma}: \gamma \in N\right)$ is called a $\Delta$-system [1] if $A_{\mu} \cap A_{\gamma}=A_{\rho} \cap A_{\sigma}$ whenever $\mu, \gamma, \rho, \sigma \in N ; \mu \neq \gamma ; \rho \neq \sigma$. If we want to indicate the cardinality $|N|$ of the index set $N$ then we speak of a $\Delta(|N|)$ system. In [1] conditions on cardinals $a, b, c$ were obtained which imply that every $(a, b)$-system contains a $\Delta(c)$-subsystem. In [2], for every choice of cardinals $b, c$ such that

$$
b \geqq 2 ; c \geqq 3 ; b+c \geqq \aleph_{0}
$$

the least cardinal $a=f_{\Delta}(b, c)$ was determined which has the property that

$$
\text { every }(a,<b) \text {-system contains a } \Delta(c) \text {-subsystem. }
$$

Let $b^{+}$be the least cardinal greater than $b$. It is easy to see that the following two statements are equivalent:

$$
\begin{gathered}
\text { every }\left(a,<b^{+}\right) \text {-system contains a } \Delta(c) \text {-subsystem, } \\
\text { every }(a, b) \text {-system contains a } \Delta(c) \text {-subsystem. }
\end{gathered}
$$

In the present note we prove a best possible theorem (Theorem 1) on the size of the largest $\Delta$-subsystem that can be found in every $\left(m^{+}, m\right)$-system $\left(A_{\gamma}\right.$ : $\gamma \in N$ ) which satisfies $\left|A_{\mu} \cap A_{\gamma}\right|<n$ for $\mu, \gamma \in N ; \mu \neq \gamma$. Here $m \geqq \aleph_{0}$, and $n$ is a given cardinal, $n<m$. In proving this theorem the authors have received valuable help from A. Hajnal.

We now introduce a condition on systems of sets which is less exacting than that of being a $\Delta$-system. The system $\left(A_{\gamma}: \gamma \in N\right)$ is called a weak $\Delta$-system ( $w k$ 
$\Delta$-system) if

$$
\left|A_{\mu} \cap A_{\gamma}\right|=\left|A_{\rho} \cap A_{\sigma}\right|
$$

whenever $\mu, \gamma, \rho, \sigma \in N ; \mu \neq \gamma ; \rho \neq \sigma$.

To avoid misunderstandings we shall henceforth replace the term " $\Delta$-system" by "strong $\Delta$-system (st $\Delta$-system). Clearly, every st $\Delta$-system is also a wk $\Delta$-system, and the system $(\{1,2\},\{1,3\},\{2,3\})$ is weak but not strong. In Theorem 2 we give an implication in the opposite direction. For cardinals $a, b, c$, let the relation

$$
(a, b) \rightarrow \text { wk } \Delta(c)
$$

mean that every $(a, b)$-system contains a wk $\Delta(c)$-subsystem, and similarly for the relation

$$
(a, b) \rightarrow \text { st } \Delta(c) \text {. }
$$

The negation of a relation involving an arrow $\rightarrow$ is obtained by writing $\rightarrow$ instead of $\rightarrow$. The symbol wk $\Delta$ by itself denotes the class of all wk $\Delta$-systems, and similarly in other cases, such as st $\Delta(c)$.

In Section 5 we prove a number of results on $\Delta$-systems. In Section 7 we give a complete discussion of the relation (1) for $a, b \geqq \aleph_{0}$. In this discussion, as well as in some of our theorems, we shall assume the generalised continuum hypothesis $(\mathrm{GCH})$.

\section{Terminology and notation}

Roman capitals denote sets, and $A \subset B$ denotes inclusion in the wide sense. For every system $\left(A_{\gamma}: \gamma \in N\right)$ and $M \subset N$, we put $A_{M}=\bigcup(\gamma \in M) A_{\gamma}$. The system $\left(A_{\gamma}: \gamma \in N\right)$ is called an $(a, b)$-system if $|N|=a$ and $\left|A_{\gamma}\right|=b$ for all $\gamma \in N$. The class of all $(a, b)$-systems is denoted by $\Omega(a, b)$. For every set $A$ and every cardinal $r$ we put

$$
[A]^{r}=\{X \subset A:|X|=r\} .
$$

For cardinals $a, c, d, r$ the partition relation

$$
a \rightarrow(c)_{d}^{r}
$$

means that whenever $A$ and $D$ are sets; $|A|=a ;|D|=d ;[A]^{r}=\cup(\lambda \in D) I_{\lambda}$ then there is a set $A^{\prime} \in[A]^{c}$ and an element $\lambda$ of $D$ such that $\left[A^{\prime}\right]^{r} \subset I_{\lambda}$. For every cardinal $m$ we put $m^{+}=\min \{n: n>m\}$. If $m$ has the form $p^{+}$then we put $m^{-}=p$, and in all other cases $m^{-}=m$. By $\omega(m)$ we denote the least ordinal $\lambda$ such that $|\lambda|=m$. For every ordinal $\alpha$, put $\underline{\alpha}=\{\lambda: \lambda<\alpha\}$, and for every cardinal $m$ put $\underline{m}=\omega(m)$. If $m \geqq \aleph_{0}$, then the symbol $\mathrm{cf}(m)$ denotes the least

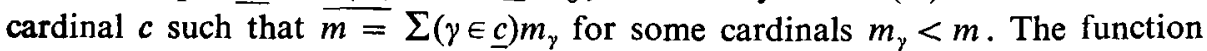
cf is the cofinality function. Instead of $(\operatorname{cf}(m))^{+}$we write $\mathrm{cf}^{+}(m)$, and similarly 
in other cases. For objects $x, y$ the symbol $\{x, y\}_{\neq}$denotes the set $\{x, y\}$ and at the same time expresses the condition that $x \neq y$. If $d$ is a cardinal then the symbol $\left(A_{\gamma}: \gamma \in N\right)_{d}$ denotes the system $\left(A_{\gamma}: \gamma \in N\right)$ and expresses the condition that $\left|A_{\mu} \cap A_{\gamma}\right|=d$ for $\{\mu, \gamma\}_{\neq} \subset N$. Symbols like $\left(A_{\gamma}: \gamma \in N\right)_{<d}$ have the obvious meaning.

We use the obliterator ${ }^{\wedge}$; its effect consists in deleting from a well-ordered sequence the element above which it is placed. Other uses of ${ }^{\wedge}$ will be self-explanatory. If $x=\left(x_{0}, \cdots, \hat{x}_{k}\right)$ and $y=\left(y_{0}, \cdots, \hat{y}_{k}\right)$ are sequences of the same length $k$, and $x \neq y$, then there is an ordinal $i<k$, denoted by $x \circ y$, such that $x_{j}=y_{j}$ $(j<i) ; x_{i} \neq y_{i}$. We shall occasionally use that

$$
\begin{gathered}
\left\{j<k:\left(x_{0}, \cdots, \hat{x}_{j}\right)=\left(y_{0}, \cdots, \hat{y}_{j}\right)\right\}=\underline{x \circ y+1,} \\
\left\{j<k:\left(x_{0}, \cdots, x_{j}\right)=\left(y_{0}, \cdots, y_{j}\right)\right\}=\underline{x} \circ y .
\end{gathered}
$$

If $(S, \prec)$ is an ordered set and $n$ is an ordinal; $x_{0}, \cdots, \hat{x}_{n} \in S$, then the symbol $\left\{x_{0}, \cdots, \hat{x}_{n}\right\}_{<}$denotes the set $\left\{x_{0}, \cdots, \hat{x}_{n}\right\}$ and expresses the condition that $x_{\mu} \prec x_{y}$ for $\mu<\gamma<n$. A set $A \subset S$ is said to be cofinal in $(S, \prec)$ if $\bigcup(x \in A)$ $\{y \in S: y \preccurlyeq x\}=S$. It is well known that if $a \geqq \aleph_{0}$ and $\operatorname{tp}(S, \prec)=\omega(a)$, then cf $(a)$ is the minimum of the cardinals of the sets $A$ which are cofinal in $(S, \prec)$. Finally, a symbol such as $\left(\left(A_{\gamma}\right)_{\gamma \in N}, B\right)$ denotes the family $\left(D_{\lambda}: \lambda \in L\right)$, where $L=N \cup\{\rho\} ; \rho \notin N ; D_{\lambda}=A_{\lambda}$ for $\lambda \in N$, and $D_{\rho}=B$.

\section{3.}

THEOREM 1. Let $m, n$ be cardinals; $m \geqq \aleph_{0} ; n<m$. Let $\mathscr{F}=\left(A_{\gamma}: \gamma \in N\right)_{<n} \in$ $\Omega\left(m^{+}, m\right)$.

(i) If $m^{n}=m$ then the system $\mathscr{F}$ has a st $\Delta\left(m^{+}\right)$-subsystem;

(ii) If $m^{n}>m$ and $\mathrm{GCH}$ holds, then $\mathscr{F}$ has a st $\Delta(p)$-subsystem for every $p<m$;

(iii) the proposition (ii) becomes false if the hypothesis $p<m$ is replaced by $p \leqq m$.

Remarks. (a) A. Hajnal made valuable contributions towards proving Theorem 1.

(b) It is well known that, for every $m \geqq \aleph_{0}$, the relation $m^{n}=m$ holds if and only if $1 \leqq n<\mathrm{cf}(m)$ (assuming GCH).

\section{Discretization sequences}

Let $\mathscr{F}=\left(A_{\gamma}: \gamma \in N\right)$ be a given system. A discretization sequence ( $d$-sequence $)$ of $\mathscr{F}$ is any sequence $\left(N_{0}, \cdots, \hat{N}_{k}\right)$ such that $k=\omega\left(|N|^{+}\right)$and, for each $\lambda<k$, the set $N_{\lambda}$ is maximal with the properties

$$
N_{\lambda} \subset N-N_{\underline{\lambda}} ;\left(A_{\gamma}-A_{\underline{\underline{\lambda}}}: \gamma \in N_{\lambda}\right)_{0} .
$$


Thus $N_{0}$ is maximal such that $N_{0} \subset N ;\left(A_{\gamma}: \gamma \in N_{0}\right)_{0}$. Next,

$N_{1}$ is maximal such that $N_{1} \subset N-N_{0} ;\left(A_{\gamma}-A_{N_{0}}: \gamma \in N_{1}\right)_{0}$;

$N_{2}$ is maximal such that $N_{2} \subset N-\left(N_{0} \cup N_{1}\right) ;\left(A_{\gamma}-A_{N_{0}} \cup N_{1}: \gamma \in N_{2}\right)_{0}$, and so on. Let us put $A_{N \underline{\lambda}}=S_{\lambda}$ for every ordinal $\lambda<k$, and $A_{N_{\underline{p}}}=S_{p}$ for every cardinal $p<|k|$.

LEMMA 1. Let $\left(N_{0}, \cdots, \hat{N}_{k}\right)$ be a d-sequence of $\left(A_{\gamma}: \gamma \in N\right)$.

There is $k_{0}<k$ such that $\left\{\lambda<k: N_{\lambda} \neq \varnothing\right\}=\underline{k}_{0}$;

if $\lambda<k ;\{\mu, \gamma\}_{\neq} \subset N_{\lambda}$, then $A_{\mu} \cap A_{\gamma} \subset S_{\lambda}$;

if $\lambda<k ; \mu \in N-N_{\underline{\lambda+1}}$, then $A_{N_{\lambda}} \cap A_{\mu} \notin S_{\lambda}$;

if $\lambda<k ; \mu \in N-N_{\underline{\lambda}}$, then $\left|S_{\lambda} \cap A_{\mu}\right| \geqq|\lambda|$.

Proof of (3). Let $\lambda<\mu<k ; N_{\lambda}=\varnothing$. Then, by definition of $N_{\mu}$, we have $N_{\mu}=\varnothing$. Also, $|k|>|N|$.

Proof of (4). $A_{\mu} \cap A_{\gamma}-S_{\lambda}=\left(A_{\mu}-S_{\lambda}\right) \cap\left(A_{\gamma}-S_{\lambda}\right)=\varnothing$ by definition of $N_{\lambda}$.

Proof of (5). The relation $\left(A_{\gamma}-S_{\lambda}: \gamma \in N_{\lambda} \cup\{\mu\}\right)_{0}$ is false by the maximality of $N_{\lambda}$. Hence there is $\gamma \in N_{\lambda}$ such that $\left(A_{\mu}-S_{\lambda}\right) \cap\left(A_{\gamma}-S_{\lambda}\right) \neq \varnothing$. Then $A_{\mu} \cap$ $\cap A_{\gamma} \notin S_{\lambda} ; A_{\mu} \cap A_{N \lambda} \supset A_{\mu} \cap A_{\gamma} \notin S_{\lambda}$.

Proof of (6). Let $\kappa<\lambda$. Then $\mu \in N-N_{\lambda} \subset N-N_{\kappa+1}$ and, by (5), there is $x_{\kappa} \in A_{N_{k}} \cap A_{\mu}-S_{\kappa}$. If $\kappa^{\prime}<\kappa$ then $x_{\kappa} \in A_{N}-\overline{A_{N_{k}}} \subset A_{N}-\left\{x_{\kappa^{\prime}}\right\}$. Hence $\left|S_{\lambda} \cap A_{\mu}\right| \geqq\left|\left\{x_{0}, \cdots, \hat{x}_{\lambda}\right\}_{\neq}\right|=|\lambda|$. This proves Lemma 1 .

\section{ProOF OF ThEOREM 1.}

Proof of (i). Let $\left(N_{0}, \cdots, \hat{N}_{k}\right)$ be a $d$-sequence of $\mathscr{F}$. Then $k=\omega\left(m^{++}\right)$.

CASE 1. There is $\kappa \in \underline{n}$ with $\left|N_{\kappa}\right|=m^{+}$. Then there is $\kappa_{0}=\min \left\{\kappa \in n:\left|N_{\kappa}\right|\right.$ $\left.=m^{+}\right\}$. Then $\left|S_{\kappa_{0}}\right| \leqq n \bar{m} m=m$. Put $P=\left\{\gamma \in N_{\kappa_{0}}:\left|A_{\gamma} \cap S_{\kappa_{0}}\right| \geqq n\right\} ; Q=N_{\kappa_{0}}$ $-P$.

CASE 1a. $|P|=m^{+}$. Then, for $\gamma \in P$, there is $B_{\gamma} \in\left[A_{\gamma} \cap S_{\kappa_{0}}\right]^{n}$. Then $\left|\left\{B_{\gamma}: \gamma \in P\right\}\right| \leqq\left|\left[S_{\kappa_{0}}\right]^{n}\right| \leqq m^{n}=m<|P|$, and there is $\{\mu, \gamma\}_{\neq} \subset P$ such that $B_{\mu}=B_{\gamma}$. Then $\left|A_{\mu} \cap A_{\gamma}\right| \geqq\left|B_{\mu} \cap B_{\gamma}\right|=\left|B_{\mu}\right|=n>\left|A_{\mu} \cap A_{\gamma}\right|$ which is a contradiction.

CASE 1b. $|P| \leqq m$. Then $|Q|=m^{+} ;\left|A_{\gamma} \cap S_{\kappa_{0}}\right|<n(\gamma \in Q)$. Since $\left|\left[S_{\kappa_{0}}\right]^{<n}\right|$ $\leqq \sum(t<n) m^{t} \leqq n m^{n}=m$, there is $D \in\left[S_{\kappa_{0}}\right]^{<n}$ and $Q^{\prime} \in[Q]^{m+}$ such that $A_{\gamma} \cap S_{\kappa_{0}}$ $=D$ for all $\gamma \in Q^{\prime}$. Then, by Lemma $1(4), A_{\mu} \cap A_{\gamma}=D$ for $\{\mu, \gamma\}_{\neq} \subset Q^{\prime}$ and so

$$
\left(A_{\gamma}: \gamma \in Q^{\prime}\right) \in \text { st } \Delta\left(m^{+}\right) \text {. }
$$


CASE 2. $\left|N_{\kappa}\right| \leqq m(\kappa \in n)$. Then $\left|N_{n}\right| \leqq n m=m ;\left|N-N_{\underline{n}}\right|=m^{+}$. By Lemma $1(6),\left|A_{\gamma} \cap S_{n}\right| \geqq n\left(\bar{\gamma} \in N-N_{\underline{n}}\right)$. Choose $B_{\gamma} \in\left[A_{\gamma} \cap S_{n}\right]^{n}$ for $\gamma \in N-N_{\underline{n}}$. Then

$$
\left|\left\{B_{\gamma}: \gamma \in N-N_{n}\right\}\right| \leqq\left|\left[S_{n}\right]^{n}\right| \leqq(m m)^{n}=m<\left|N-N_{n}\right|,
$$

and there is $\{\mu, \gamma\}_{\neq} \subset N-N_{\underline{n}}$ such that $B_{\mu}=B_{\gamma}$. Then

$$
\left|A_{\mu} \cap A_{\gamma}\right| \geqq\left|B_{\mu} \cap B_{\gamma}\right|=\left|B_{\mu}\right|=n>\left|A_{\mu} \cap A_{\gamma}\right|
$$

which is a contradiction. This proves (i).

Before proving (ii) we establish a lemma.

LEMMA 2. Let

$$
\begin{array}{cl}
n<m \geqq \aleph_{0} ; & m^{n}>m ;|S|=m ;|N|=m^{+} ; \\
& X_{\gamma} \in[S]^{m}(\gamma \in N) .
\end{array}
$$

Assume GCH. Then there is $\{\mu, \gamma\}_{\neq} \subset N$ such that $\left|X_{\mu} \cap X_{\gamma}\right|>n$.

Proof of Lemma 2. $n \geqq \operatorname{cf}(m)$. There is a respresentation $S=T_{0} \cup \cdots \cup \hat{T}_{t}$ such that $t=\omega(\operatorname{cf}(m)) ;\left|T_{\lambda}\right|=m_{\lambda}<m(\lambda<t)$. Let $\gamma \in N$. Then there is $\lambda_{\gamma}<t$ such that $\left|X_{y} \cap T_{\lambda_{y}}\right|>n$. For otherwise we obtain the contradiction

$$
m=\left|X_{\gamma}\right| \leqq \sum(\lambda<t)\left|X_{\gamma} \cap T_{\lambda}\right| \leqq|t| n<m .
$$

Now there is $M \in[N]^{m^{+}}$and $\lambda^{\prime}$ such that $\lambda_{\gamma}=\lambda^{\prime}(\gamma \in M)$. Then

$$
\left|X_{\gamma} \cap T_{\lambda^{\prime}}\right|>n(\gamma \in M) \text {. }
$$

Since $\left|\left[T_{\lambda^{\prime}}\right]^{>n}\right| \leqq 2^{m_{\lambda^{\prime}}}<m^{+}$, there is $\{\mu, \gamma\}_{\neq} \subset M$ with $X_{\mu} \cap T_{\lambda^{\prime}}=X_{\gamma} \cap T_{\lambda^{\prime}}$. Then $\left|X_{\mu} \cap X_{\gamma}\right| \geqq\left|X_{\mu} \cap X_{\gamma} \cap T_{\lambda^{\prime}}\right|=\left|X_{\mu} \cap T_{\lambda^{\prime}}\right|>n$.

Proof of Theorem 1 (ii). Let $\left(N_{0}, \cdots, \hat{N}_{k}\right)$ be a $d$-sequence of $\left(A_{\gamma}: \gamma \in N\right)$. Then $k=\omega\left(m^{++}\right)$. Let $S_{\lambda}$ and $S_{p}$ have their previous meaning.

CASE 1. $\left|N_{m}\right| \leqq m$. Then $\left|N-N_{m}\right|=m^{+} ;\left|S_{m}\right| \leqq m$. By Lemma $1(6)$,

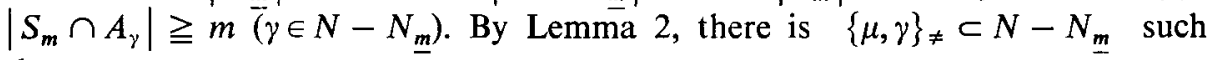
that

$$
\left|A_{\mu} \cap A_{\gamma}\right| \geqq\left|\left(S_{m} \cap A_{\mu}\right) \cap\left(S_{m} \cap A_{\gamma}\right)\right|>n>\left|A_{\mu} \cap A_{\gamma}\right|
$$

which is false.

CASE 2. $\left|N_{m}\right|=m^{+}$. Then there is $\lambda_{0}=\min \left\{\lambda \in \underline{m}:\left|N_{\lambda}\right|=m^{+}\right\}$. Then

$$
\left|A_{\gamma} \cap S_{\lambda_{0}}\right| \leqq\left|S_{\lambda_{0}}\right| \leqq m(\gamma \in N) \text {. }
$$

CASE 2a. There is $M \in\left[N_{\lambda_{0}}\right]^{m^{+}}$such that $\left|A_{\gamma} \cap S_{\lambda_{0}}\right|=m(\gamma \in M)$. Then, by Lemma 2, there is $\{\mu, \gamma\}_{\neq} \subset M$ such that 


$$
\left|\left(A_{\mu} \cap S_{\lambda_{0}}\right) \cap\left(A_{\gamma} \cap S_{\lambda_{0}}\right)\right|>n>\left|A_{\mu} \cap A_{\gamma}\right| .
$$

This is a contradiction.

CASE $2 b$. There is $M \in\left[N_{\lambda_{0}}\right]^{m^{+}}$such that $\left|A_{\gamma} \cap S_{\lambda_{0}}\right|<m(\gamma \in M)$.

Then there is $M^{\prime} \in[M]^{m^{+}}$such that the cardinal $\left|A_{\gamma} \cap S_{\lambda_{0}}\right|$ is constant for $\gamma \in M^{\prime}$, say $\left|A_{\gamma} \cap S_{\lambda_{0}}\right|=q\left(\gamma \in M^{\prime}\right)$. There are sets $X_{\gamma}, B_{\gamma}$ such that $\left(\left(X_{\gamma}\right)_{\gamma \in M^{\prime}}, A_{N}\right)_{0}$ and $\left|B_{\gamma}\right|=p+q=p_{0}$, say $\left(\gamma \in M^{\prime}\right)$, where $B_{\gamma}=\left(A_{\gamma} \cap S_{\lambda_{0}}\right) \cup X_{\gamma}\left(\gamma \in M^{\prime}\right)$. Then $\left(B_{\gamma}: \gamma \in M^{\prime}\right) \in \Omega\left(\geqq p_{0}^{++}, p_{0}\right)$, and by [1], Theorem I, there is $M^{\prime \prime} \subset M^{\prime}$ such that $\left(B_{\gamma}: \gamma \in M^{\prime \prime}\right) \in$ st $\Delta\left(p_{0}^{++}\right)$. Then $\left(A_{\gamma} \cap S_{\lambda_{0}}: \gamma \in M^{\prime \prime}\right) \in$ st $\Delta\left(p_{0}^{++}\right)$and, by Lemma 1, $\left(A_{\gamma}: \gamma \in M^{\prime \prime}\right) \in$ st $\Delta\left(p_{0}^{++}\right)$. This proves Theorem 1 (ii).

ProOF OF THEOREM 1 (iii). It suffices to find a system

$$
\left(A_{\gamma}: \gamma \in N\right)_{<\operatorname{cf}(m)} \in \Omega\left(m^{+}, m\right)
$$

which has no st $\Delta(m)$-subsystem. Put $k=\omega(\operatorname{cf}(m))$. There are cardinals $m_{\lambda}$ such that $m_{0}, \cdots, \hat{m}_{k}<m=m_{0}+\cdots+\hat{m}_{k}$. Put

$$
\begin{gathered}
N=\left\{\gamma=\left(\gamma_{0}, \cdots, \hat{\gamma}_{k}\right): \gamma_{\lambda} \in \underline{m}_{\lambda}(\lambda<k)\right\}, \\
B_{\gamma}=\left\{\left(\gamma_{0}, \cdots, \hat{\gamma}_{\lambda}\right): \lambda<k\right\} \quad\left(\gamma=\left(\gamma_{0}, \cdots, \hat{\gamma}_{k}\right) \in N\right) .
\end{gathered}
$$

Then $\left(B_{\gamma}: \gamma \in N\right) \in \Omega\left(\Pi m_{\lambda},|k|\right)$. We have $\Pi m_{\lambda}=m^{+} ;|k|=\operatorname{cf}(m)<m$. Let $\left|X_{\gamma}\right|=m(\gamma \in N)$ and $\left(\left(X_{\gamma}\right)_{y \in N}, B_{N}\right)_{0}$, and put $A_{\gamma}=B_{\gamma} \cup X_{\gamma}(\gamma \in N)$. Then $\left(A_{\gamma}: \gamma \in N\right) \in \Omega\left(m^{+}, m\right)$. Let $\{\mu, \gamma\}_{\neq} \subset N$. Then there is $\lambda_{0}=\mu \circ \gamma$, and we have

$$
\left|A_{\mu} \cap A_{\gamma}\right|=\left|\left(B_{\mu} \cup X_{\mu}\right) \cap\left(B_{\gamma} \cup X_{\gamma}\right)\right|=\left|B_{\mu} \cap B_{\gamma}\right|=\left|\lambda_{0}\right|<|k|=\operatorname{cf}(m) .
$$

Now let $M \subset N$ and $\left(A_{\gamma}: \gamma \in M\right) \in$ st $\Delta$. Then $\left(B_{\gamma}: \gamma \in M\right) \in$ st $\Delta$. But then there is $\lambda_{1}<k$ such that $\mu \circ \gamma=\lambda_{1}$ and $B_{\mu} \cap B_{\gamma}=\left\{\left(\rho_{0}, \cdots, \hat{\rho}_{\lambda}\right): \lambda \leqq \lambda_{1}\right\}$ for all $\{\mu, \gamma\}_{\neq} \subset M$. Here $\rho_{\lambda} \in \underline{m}_{\lambda}\left(\lambda<\lambda_{1}\right)$, and $\rho_{0}, \cdots, \hat{\rho}_{\lambda_{1}}$ are independent of $\mu, \gamma$. Therefore

$$
|M|=\left|\left\{\gamma_{\lambda_{1}}:\left(\gamma_{0}, \cdots, \hat{\gamma}_{k}\right) \in M\right\}\right| \leqq m_{\lambda_{1}}<m,
$$

and the proof of Theorem 1 is completed.

\section{Some special Theorems}

THEOREM 2. Let $\left(A_{\gamma}: \gamma \in N\right) \in \mathrm{wk} \Delta$. Assume that

(i) $\left|A_{\gamma}\right| \leqq n<\aleph_{0}$ for $\gamma \in N$,

(ii) $\left|A_{\mu} \cap A_{\gamma}\right|=k$ for $\{\mu, \gamma\}_{\neq} \subset N$,

(iii) $|N|>1+n\left(\begin{array}{c}n \\ k\end{array}\right)$.

Then $\left(A_{\gamma}: \gamma \in N\right) \in$ st $\Delta$.

PRoof. Let $\gamma_{0} \in N$. By (i) and (ii),

$$
\left|\left\{A_{\gamma} \cap A_{\gamma_{0}}: \gamma \in N-\left\{\gamma_{0}\right\}\right\}\right| \leqq\left(\begin{array}{l}
n \\
k
\end{array}\right) \text {. }
$$


Hence, by (iii), there are sets $M, D$ with $M \in\left[N-\left\{\gamma_{0}\right\}\right]^{n+1}$ and $D \in\left[A_{\gamma_{0}}\right]^{k}$ such that $A_{\mu} \cap A_{\gamma_{0}}=D$ for $\mu \in M$.

CASE 1. There is $\gamma_{1} \in N-\left\{\gamma_{0}\right\}$ with $D \notin A_{\gamma_{1}}$. Then, for every $\mu \in M$, we have $A_{\mu} \cap A_{\gamma_{1}} \neq D$, and there is $x_{\mu} \in A_{\mu} \cap A_{\gamma_{1}}-D$. Then

$$
\left|\left\{x_{\mu}: \mu \in M\right\}\right| \leqq\left|A_{\gamma_{1}}\right| \leqq n<|M| \text {, }
$$

and there is $\{\rho, \sigma\}_{\neq} \subset M$ with $x_{\rho}=x_{\sigma}$. Then $x_{\rho} \in A_{\rho} \cap A_{\sigma}=D$ which is a contradiction.

CASE 2. $D \subset A_{\gamma}$ for all $\gamma \in N-\left\{\gamma_{0}\right\}$. Then $A_{\mu} \cap A_{\gamma}=D$ for $\{\mu, \gamma\}_{\neq} \subset N$ and the theorem follows.

Definitions: $\left(A_{\gamma}: \gamma \in N\right)$ is called a system without repetition if $A_{\mu} \neq A_{\gamma}$ for $\{\mu, \gamma\}_{\neq} \subset N$. For $n<\aleph_{0}$, denote by $g(n)$ the largest integer such that there exists a $(g(n), n)$-system without repetition which has no wk $\Delta(3)$-subsystem. Let $h(n)$ be defined similarly but with repetitions allowed.

It is easy to see that $g(1)=1 ; g(2)=5 ; g(3) \geqq 10$. D. Hanson proved that $g(3)=10$.

THEOREM 3. For all $n$ with $0<n<\aleph_{0}$,

$$
\text { (i) } h(n)=2 g(n), \quad \text { (ii) } g(n+1) \geqq 2 g(n) \text {. }
$$

COROLlaRY. $g(n) \geqq 5.2^{n-2}$ for $n \geqq 2$.

ProOF of (i). If $\left(A_{1}, A_{2}, \cdots, A_{x}\right)$ is a $(g(n), n)$-system without repetition which has no wk $\Delta(3)$-subsystem, then $\left(A_{1}, \cdots, A_{x}, A_{1}, \cdots, A_{x}\right)$ is a $(2 g(n), n)$-system, with repetition, and again without wk $\Delta(3)$-subsystem. Hence $h(n) \geqq 2 g(n)$. If, for some $n$, we have $h(n)>2 g(n)$ then there is a $(>2 g(n), n)$-system without wk $\Delta(3)$ subsystem. Such a system contains at least $g(n)+1$ distinct members, and these form a system whose existence contradicts the definition of $g(n)$. Hence (i).

Proof OF (ii). There is a $(g(n), n)$-system $\left(A_{\gamma}: \gamma \in N\right)$ without repetition and without wk $\Delta(3)$-subsystem. Let $x_{\gamma \lambda}$ be any $2 g(n)$ distinct objects, for $\gamma \in N$ and $\lambda \in \underline{\mathbf{2}}$ which do not belong to $A_{N}$. Then it is easily verified that

$$
\left(A_{\gamma} \cup\left\{x_{\gamma \lambda}\right\}: \gamma \in N ; \lambda \in \underline{2}\right)
$$

is a $(2 g(n), n+1)$-system without repetition and without wk $\Delta(3)$-subsystem. This proves (ii).

THEOREM 4. Let $a>0$ and $1 \leqq n \leqq \aleph_{0}$. Then there is an $\left(a^{n}, n\right)$-system $\left(A_{x}: x \in X\right)_{<n}$ which has no wk $\Delta\left(a^{+}\right)$-subsystem.

ProOf. Put $X=\left\{x=\left(x_{0}, \cdots, \hat{x}_{n}\right): x_{0}, \cdots, \hat{x}_{n} \in \underline{a}\right\}$;

$$
A_{x}=\left\{\left(x_{0}, \cdots, x_{\gamma}\right): y \in \underline{n}\right\} \quad(x \in X) \text {. }
$$


Then $\left(A_{x}: x \in X\right)_{<n} \in \Omega\left(a^{n}, n\right)$. If $\{x, y\}_{\neq} \subset X$ then

$$
\left|A_{x} \cap A_{y}\right|=\left|\left\{\left(x_{0}, \cdots, x_{\gamma}\right): \gamma<x \circ y\right\}\right|=x \circ y<n \text {. }
$$

Let $X^{\prime} \subset X$ and $\left(A_{x}: x \in X^{\prime}\right) \in$ wk $\Delta$. Then there is $m<n$ such that $x \circ y=m$ for $\{x, y\}_{\neq} \subset X^{\prime}$, and hence $\left|X^{\prime}\right|=\left|\left\{x_{m}: x \in X^{\prime}\right\}\right| \leqq a$. The theorem follows.

THEOREM 5. Let $\alpha$ be a non-zero ordinal, and put $d_{\alpha}=2^{|\alpha|}$. Then there is $a\left(d_{\alpha}, \aleph_{\alpha}\right)$-system $\left(A_{\gamma}: \gamma \in N\right)_{<\aleph_{\alpha}}$ without wk $\Delta(3)$-subsystem. In particular, we have $\left(d_{\alpha}, \aleph_{\alpha}\right) \rightarrow$ wk $\Delta(3)$. If (i) $2^{|\beta|} \leqq \aleph_{\alpha}$ for $\beta<\alpha$, (ii) $\aleph_{\alpha}=|\alpha|$, then we can stipulate that, in addition, $\left|A_{N}\right|=\aleph_{\alpha}$.

REMARK. The condition (i) is a weak version of the generalized continuum hypothesis, and the condition (ii) is equivalent to $\omega_{\alpha}=\alpha$ and is known to hold for some $\alpha$.

Proof. Let the letter $\lambda$ denote elements of 2 , and the letters $\beta, \gamma, \delta$ elements of $\underline{\alpha}$. Let $\left|X\left(\lambda_{0}, \cdots, \lambda_{\beta}\right)\right|=\aleph_{\beta+1}$ for all $\beta, \lambda_{0}, \cdots, \lambda_{\beta}$, and

$$
\left(X\left(\lambda_{0}, \cdots, \lambda_{\beta}\right): \beta \in \underline{\alpha} ; \lambda_{0}, \cdots, \lambda_{\beta} \in \underline{2}\right)_{0} .
$$

Put $N=\left\{\left(\lambda_{0}, \cdots, \hat{\lambda}_{\alpha}\right): \lambda_{0}, \cdots, \hat{\lambda}_{\alpha} \in 2\right\}$ and $A\left(\lambda_{0}, \cdots, \hat{\lambda}_{\alpha}\right)=\bigcup(\beta<\alpha) X\left(\lambda_{0}, \cdots, \lambda_{\beta}\right)$ for $\left(\lambda_{0}, \cdots, \hat{\lambda}_{\alpha}\right) \in N$. Then $|N|=2^{|\alpha|} ;\left|A\left(\lambda_{0}, \cdots, \hat{\lambda}_{\alpha}\right)\right|=\sum(\beta<\alpha) \aleph_{\beta+1}=\aleph_{\alpha}$. Now suppose that $\left\{\left(\lambda_{0}, \cdots, \hat{\lambda}_{\alpha}\right),\left(\lambda_{0}^{\prime}, \cdots, \hat{\lambda}_{\alpha}^{\prime}\right),\left(\lambda_{0}^{\prime \prime}, \cdots, \hat{\lambda}_{\alpha}^{\prime \prime}\right)\right\}_{\neq} \subset N$. Put $\rho=\lambda \circ \lambda^{\prime}$. Then $\left|A(\lambda) \cap A\left(\lambda^{\prime}\right)\right|=\Sigma(\gamma<\rho) \aleph_{\gamma+1} \leqq \aleph_{\rho}<\aleph_{\alpha}$. Put $\sigma=\lambda \circ \lambda^{\prime \prime} ; \tau=\lambda^{\prime} \circ \lambda^{\prime \prime}$. Change the notation, if necessary, so that $\rho \leqq \sigma \leqq \tau$. Then

$\rho<\tau ;\left|A(\lambda) \cap A\left(\lambda^{\prime}\right)\right| \leqq \aleph_{\rho}<\aleph_{\rho+1} \leqq \aleph_{\tau}=\sum(\gamma<\tau) \aleph_{\gamma+1}=\left|A\left(\lambda^{\prime}\right) \cap A\left(\lambda^{\prime \prime}\right)\right|$.

Hence the $\left(2^{|\alpha|}, \aleph_{\alpha}\right)$-system $(A(\lambda): \lambda \in N)_{<\aleph_{\alpha}}$ has no wk $\Delta(3)$-subsystem. Now suppose that (i) and (ii) hold. Then

$$
\begin{aligned}
|\bigcup(\lambda \in N) A(\lambda)| & =\left|\bigcup\left(\beta<\alpha ; \lambda_{0}, \cdots, \lambda_{\beta} \in \underline{2}\right) X\left(\lambda_{0}, \cdots, \lambda_{\beta}\right)\right| \\
& =\Sigma(\beta<\alpha) 2^{|\beta+1|} \aleph_{\beta+1}=\aleph_{\alpha} ;|N|=2^{|\alpha|}=2^{\aleph_{\alpha}} .
\end{aligned}
$$

Hence, on changing the notation slightly, we obtain a $\left(2^{\aleph_{\alpha}}, \aleph_{\alpha}\right)$-system $\left(A_{\mu}: \mu \in M\right)$ without wk $\Delta(3)$-subsystem, and now $\left|A_{M}\right|=\aleph_{\alpha}$.

THEOREM 6. Let $a=\aleph_{\omega}$. Then (i) assuming GCH, there is an $\left(a^{+}, \aleph_{0}\right)$ system $\left(A_{\lambda}: \lambda \in L\right)_{<\aleph_{0}}$ with $\left|A_{L}\right| \leqq a ;(i i)$ no $\left(a^{+}, \aleph_{0}\right)$-system $\left(B_{\lambda}: \lambda \in L\right)<\aleph$.. with $\left|B_{L}\right| \leqq a$ has $a$ wk $\Delta\left(a^{+}\right)$-subsystem; (iii) if GCH holds then

$$
\left(\aleph_{\omega+1}, \aleph_{0}\right) \rightarrow \text { wk } \Delta\left(\aleph_{\omega+1}\right)
$$


Remarks. The result (i) is due to A. Tarski. For the convenience of the reader we give a proof. In Section 7, Case 1 b2a1, we prove $\left(\aleph_{\omega+1}, \aleph_{0}\right) \rightarrow w k \Delta\left(\aleph_{\omega}\right)$, a relation which is stronger than (iii).

Proof of (i). Let $L$ be the set of all sequences $\lambda=\left(l_{0}, \cdots, \hat{l}_{\omega}\right)$ such that $l_{\gamma} \in \underline{\omega}_{\gamma}$ for $\gamma<\omega$. Put $A_{\lambda}=\left\{\left(l_{0}, \cdots, \hat{l}_{\mu}\right): \mu<\omega\right\}$ for $\lambda \in L$. Then $\left(A_{\lambda}: \lambda \in L\right) \in \Omega\left(a^{+}, \aleph_{0}\right)$;

$$
\left|A_{L}\right|=\mid\left\{\left(l_{0}, \cdots, \hat{l}_{\mu}\right): \mu<\omega ; l_{\gamma} \in \underline{\omega}_{\gamma} \text { for } \gamma<\mu\right\} \mid=\Sigma(\mu<\omega) \Pi(\gamma<\mu) \aleph_{\gamma}=a .
$$

If $\left\{\lambda, \lambda^{\prime}\right\}_{\neq} \subset L$ then there is $\gamma_{0}=\lambda \circ \lambda^{\prime}$, and we have $\left|A_{\lambda} \cap A_{\lambda^{\prime}}\right|=\gamma_{0}+1<\aleph_{0}$.

Proof OF (ii). Let the $\left(a^{+}, \aleph_{0}\right)$-system $\left(B_{\lambda}: \lambda \in L\right)_{<\aleph_{0}}$ satisfy $\left|B_{L}\right| \leqq a$. Let $\left(B_{\lambda}: \lambda \in L^{\prime}\right) \in$ wk $\Delta$ for some $L^{\prime} \in[L]^{a^{+}}$. Choose $\left\{\lambda^{\prime}, \lambda^{\prime \prime}\right\}_{\neq} \subset L^{\prime}$. Then $\left|B_{\lambda^{\prime}} \cap B_{\lambda^{\prime \prime}}\right|$ $=p<\aleph_{0}$. Choose $D_{\lambda} \in\left[B_{\lambda}\right]^{p+1}$ for $\lambda \in L^{\prime}$. Then $\left|\left\{D_{\lambda}: \lambda \in L^{\prime}\right\}\right| \leqq\left|B_{L}\right|<\left|L^{\prime}\right|$ and therefore there is $\{\rho, \sigma\}_{\neq} \subset L^{\prime}$ such that $D_{\rho}=D_{\sigma}$. Then

$$
p=\left|B_{\rho} \cap B_{a}\right| \geqq\left|D_{\rho}\right|=p+1
$$

which is the required contradiction.

\section{Some Lemmas}

It is convenient to use the function $\psi(a)=|\{x: x \leqq a\}|$, where $a$ ranges over cardinals. Thus, $\psi\left(\aleph_{\alpha}\right)=\aleph_{0}+|\alpha|$.

Throughout the rest of this paper we use the following notation for two fixed cardinals:

$$
a=\aleph_{\alpha} ; \quad b=\aleph_{\beta} .
$$

Furthermore, GCH is assumed without reference being made to this fact.

Lemia 3. Let $a>\operatorname{cf}(a)$. Then $(a, b) \rightarrow \mathrm{wk} \Delta(a)$.

Proof. If $n=\omega(\operatorname{cf}(a))$ then there are cardinals $a_{\gamma}$ with

$$
a_{0}, \cdots, \hat{a}_{n}<a=a_{0}+\cdots+\hat{a}_{n} .
$$

Choose sets $B_{\gamma}$ with $\left|B_{\gamma}\right|=b(\gamma<n)$ and $\left(B_{0}, \cdots, \hat{B}_{n}\right)_{0}$, and put $D_{\gamma \lambda}=B_{\gamma}$ for $\gamma<n$ and $\lambda \in \underline{a}_{\gamma}$. Then $\left(D_{\gamma \lambda}: \gamma<n ; \lambda \in \underline{a}_{\gamma}\right) \in \Omega(a, b)$. Let $D_{\gamma} \subset \underline{a}_{\gamma}(\gamma<n)$;

$$
\left(D_{\gamma \lambda}: \gamma<n ; \lambda \in D_{\gamma}\right) \in \text { wk } \Delta(c) \text {. }
$$

CASE 1. There is $\gamma_{0}<n$ such that $\left|D_{\gamma_{0}}\right| \geqq 2$. Choose $\{\sigma, \tau\}_{\neq} \subset D_{\gamma_{0}}$. Then $\left|D_{\gamma_{0} \sigma} \cap D_{\gamma_{0} \tau}\right|=b>0$. Hence $D_{\gamma}=\varnothing$ for $\gamma \in \underline{n}-\left\{\gamma_{0}\right\}$, and so

$$
c=\Sigma(\gamma<n)\left|D_{\gamma}\right|=\left|D_{\gamma_{0}}\right| \leqq a_{\gamma_{0}}<a \text {. }
$$

CASE 2. $\left|D_{\gamma}\right|<2$ for $\gamma<n$. Then $\Sigma(\gamma<n)\left|D_{\gamma}\right| \leqq|n|=\operatorname{cf}(a)<a$.

Lemma 4. Let $b<\mathrm{cf}(c)$. Then $\left(c^{+}, b\right) \rightarrow \mathrm{st} \Delta\left(c^{+}\right)$. 
PRoof. In [2], p. 471, the function $s(x, y)$ was defined for all cardinals $x, y$ such that $x \geqq 2 ; y \geqq 3 ; x+y \geqq \aleph_{0}$, by putting

$$
s(x, y)=\sup \left\{\Sigma(\gamma \in \underline{x}) y_{0} \cdots \hat{y}_{\gamma}: y_{0}, \cdots, \hat{y}_{\omega(x)}<y\right\} .
$$

We have

$$
s\left(b^{+}, c^{+}\right)=\Sigma\left(\gamma \in \underline{b}^{+}\right) c^{|\gamma|} \leqq \Sigma\left(\gamma \in \underline{b}^{+}\right) c=b^{+} c=c \leqq s\left(b^{+}, c^{+}\right) .
$$

Here, the first inequality follows from $|\gamma| \leqq b<\operatorname{cf}(c)$, and the second inequality from $b>0$. By [2], Theorem IV,

and therefore

$$
f_{\Delta}\left(b^{+}, c^{+}\right)=s^{+}\left(b^{+}, c^{+}\right),
$$

$$
\begin{gathered}
\left(s^{+}\left(b^{+}, c^{+}\right), \leqq b\right) \rightarrow \text { st } \Delta\left(c^{+}\right) ;\left(c^{+}, \leqq b\right) \rightarrow \text { st } \Delta\left(c^{+}\right) ; \\
\left(c^{+}, b\right) \rightarrow \text { st } \Delta\left(c^{+}\right) .
\end{gathered}
$$

Lemma 5. Let $a=a^{-}=\operatorname{cf}(a)>b$. Then $(a, b) \rightarrow$ st $\Delta(a)$.

PROOF. $s\left(b^{+}, a\right) \leqq \Sigma\left(\gamma \in \underline{b}^{+}\right) a^{|\gamma|} \leqq \Sigma\left(\gamma \in \underline{b}^{+}\right) a=b^{+} a=a$;

$$
s\left(b^{+}, a\right) \geqq \sup \left\{a_{0}: a_{0}<a\right\}=a .
$$

Hence $s\left(b^{+}, a\right)=a$. We now prove $f_{\Delta}\left(b^{+}, a\right)=s\left(b^{+}, a\right)$. We want to apply [2] Theorem IV (a) (iii). To do this we must prove

(i) $\aleph_{0} \leqq b^{+}<\operatorname{cf}(a) \leqq a^{-}=a$;

(ii) if $\sup \left\{a_{0}^{b}: a_{0}<a\right\}=d$ then $d=\operatorname{cf}(d)>a_{1}^{b}$ for $a_{1}<a$.

Now, (i) is true. Also,

$$
\begin{aligned}
\sup \left\{a_{0}^{b}: a_{0}<a\right\} \leqq & \sup \left\{a_{0}^{+} b^{+}: a_{0}<a\right\} \leqq a \\
& \leqq \sup \left\{a_{0}^{b}: a_{0}<a\right\} ; \sup \left\{a_{0}^{b}: a_{0}<a\right\}=a=\operatorname{cf}(a) .
\end{aligned}
$$

Finally, let $a_{1}<a$. Then $a_{1}^{b} \leqq a_{1}^{+} b^{+}<a$. This proves (ii), and we have, by [2], $f_{\Delta}\left(b^{+}, a\right)=s\left(b^{+}, a\right)=a ;\left(a,<b^{+}\right) \rightarrow$ st $\Delta(a) ;(a, b) \rightarrow$ st $\Delta(a)$.

LEMMA 6. Let $a=\operatorname{cf}(a) ; f(\mu, \gamma) \in \underline{2}$ for $\mu<\gamma \in \underline{a}^{+}$. Then there is an $\left(a^{+}, a\right)$.system $\left(F_{\gamma}: \gamma \in \underline{a}^{+}\right)$such that, for $\mu<\gamma \in \underline{a}^{+}$,

$$
\begin{aligned}
\left|F_{\mu} \cap F_{\gamma}\right|<a & \text { if } f(\mu, \gamma)=0 \\
& =a \quad \text { if } f(\mu, \gamma)=1 .
\end{aligned}
$$

Proof. 1. We begin by showing that, given any $(a, a)$-system $\left(A_{y}: \gamma \in N\right)_{<a}$, there is a set $T$ (called a $(<a)$-transversal of the system) such that 


$$
T \in\left[A_{N}\right]^{a} ; 1 \leqq\left|T \cap A_{\mu}\right|<a(\mu \in N) .
$$

We may assume $N=\underline{a}$. Then there are elements $x_{\gamma}$, for $\gamma \in \underline{a}$, such that $x_{\gamma} \in A_{\gamma}-\left(A_{\gamma} \cup\left\{x_{0}, \cdots, \hat{x}_{\gamma}\right\}\right)(\gamma \in \underline{a})$. We may put $T=\left\{x_{\gamma}: \gamma \in \underline{a}\right\}_{\neq}$. For, let $\mu \in a$. If $\xi \in T \cap A A_{\mu}$, then there is $\gamma \in \underline{a}$ such that $\xi=x_{\gamma} \in A_{y}-A_{y}$. Also, $\xi \in A_{\mu}$. Hence $\mu \notin \underline{\gamma} ; \mu \geqq \gamma$, so that $1 \leqq\left|T \cap A_{\mu}\right| \leqq\left|\left\{x_{0}, \cdots, x_{\mu}\right\}\right|=|\mu+1|<a$.

2. Choose a system $\left(S_{\alpha \beta}: \alpha \in \underline{a}^{+}: \beta \in \underline{a}\right)_{0} \in \Omega\left(a^{+}, a\right)$. We now choose sets $B_{\mu}$, for $\mu \in \underline{a}^{+}$, by the following procedure. Let $\mu_{0} \in \underline{a}^{+}$, and suppose that $B_{0}, \cdots, \hat{B}_{\mu_{0}}$ have already been defined in such a way that

$$
\left\{\begin{array}{l}
B_{\mu} \text { is a }(<a) \text {-transversal of the family } \\
\left(\left(S_{\alpha \beta}: \alpha \leqq \mu ; \beta \in \underline{a}\right), B_{0}, \cdots, \hat{B}_{\mu}\right)_{<_{a}} \text { for } \mu<\mu_{0} .
\end{array}\right.
$$

We show that

$$
\left(\left(S_{\alpha \beta}: \alpha \leqq \mu_{0} ; \beta \in \underline{a}\right), B_{0}, \cdots, \hat{B}_{\mu_{0}}\right)_{<a} .
$$

Let $\mu<\mu_{0}$. Then

$$
B_{\mu} \subset \bigcup(\alpha \leqq \mu ; \beta \in \underline{a}) S_{\alpha \beta} \cup B_{\underline{\mu}}=S_{\underline{\mu+1}, \underline{a}} \cup B_{\underline{\mu}}, \text { say. }
$$

By induction over $\mu$, we deduce that $B_{\mu} \subset S_{\underline{\mu+1}, a}\left(\mu<\mu_{0}\right)$.

(i) Let $\alpha \leqq \mu_{0} ; \beta \in \underline{a} ; \gamma<\mu_{0}$. If $\alpha \leqq \gamma$, then $\left|S_{\alpha \beta} \cap B_{\gamma}\right|<a$ by (*) with $\mu=\gamma$. If $\alpha>\gamma$, then $\left|S_{\alpha \beta} \cap B_{\gamma}\right| \leqq\left|S_{\alpha \beta} \cap S_{\underline{\gamma+1}, a}\right|=0$ since $\alpha \notin \underline{\gamma+1}$.

(ii) Let $\rho<\sigma<\mu_{0}$. Then $\left|B_{\rho} \cap B_{\sigma}\right|<a$ by $\left({ }^{*}\right)$ with $\mu=\sigma$. This proves $\left({ }^{* *}\right)$. Now let $B_{\mu_{0}}$ be a $(<a)$-transversal of the family $\left({ }^{* *}\right)$. Put $S_{\alpha}=\bigcup(\beta \in \underline{a}) S_{\alpha \beta}$ $\left(\alpha \in \underline{a}^{+}\right)$;

$$
A_{z \mu}=S_{\alpha} \cap B_{\mu}\left(\alpha \leqq \mu \in \underline{a}^{+}\right) .
$$

Then it follows, by induction on $\mu$, that

$$
B_{\mu} \subset \bigcup(\alpha \leqq \mu ; \beta \in \underline{a}) S_{\alpha \beta}=\bigcup(\alpha \leqq \mu) S_{\alpha} ;
$$

$B_{\mu}=\bigcup(\alpha \leqq \mu) S_{\alpha} \cap B_{\mu}=\bigcup(\alpha \leqq \mu) A_{\alpha \mu}\left(\mu \in \underline{a}^{+}\right)$. Since $\left|S_{\alpha \beta} \cap B_{\mu}\right| \geqq 1(\alpha \leqq \mu$ $\left.\in \underline{a}^{+} ; \beta \in \underline{a}\right)$, we have $\left|A_{\alpha \mu}\right|=a\left(\alpha \leqq \mu \in \underline{a}^{+}\right)$. Put $F_{\gamma}=S_{\gamma} \cup \cup(\mu<\gamma ; f(\mu, \gamma)$ $=1) A_{\mu \gamma}\left(\gamma \in \underline{a}^{+}\right)$. Then $S_{\gamma} \subset F_{\gamma} \subset S_{\underline{\gamma+1}}\left(\gamma \in \underline{a}^{+}\right)$;

$$
\left(F_{\gamma}: \gamma \in \underline{a}^{+}\right) \in \Omega\left(a^{+}, a\right) .
$$

Now let $\mu<\gamma \in \underline{a}^{+}$. If $f(\mu, \gamma)=1$, then $A_{\mu \gamma} \subset F_{\gamma} ; A_{\mu \gamma} \subset S_{\mu} \subset F_{\mu} ;\left|F_{\mu} \cap F_{\gamma}\right|$ $\geqq\left|A_{\mu \gamma}\right|=a$. Now suppose $f(\mu, \gamma)=0$. Then $F_{\mu} \cap F_{\gamma}=\left(S_{\mu} \cup \cup(\alpha<\mu ; f(\alpha, \mu)\right.$ $\left.=1) A_{\alpha \mu}\right) \cap\left(S_{\gamma} \cup \cup(\beta<\gamma ; f(\beta, \gamma)=1) A_{\beta \gamma}\right)$. We note that $S_{\mu} \cap S_{\gamma}=\varnothing$; if 
$f(\beta, \gamma)=1$ then $\beta \neq \mu$ and hence $S_{\mu} \cap A_{\beta \gamma} \subset S_{\mu} \cap S_{\beta}=\varnothing$. If $\alpha<\mu$, then $A_{\alpha \mu} \cap S_{\gamma} \subset S_{\alpha} \cap S_{\gamma}=\varnothing$; if $\alpha \neq \beta$, then $A_{\alpha \mu} \cap A_{\beta \gamma} \subset S_{\alpha} \cap S_{\beta}=\varnothing$. All this shows that $F_{\mu} \cap F_{\gamma} \subset \bigcup(\alpha<\mu) A_{\alpha \mu} \cap A_{\alpha \gamma} \subset B_{\mu} \cap B_{\gamma} ;\left|F_{\mu} \cap F_{\gamma}\right| \leqq\left|B_{\mu} \cap B_{\gamma}\right|<a$. This proves Lemma 6.

Lemma 7. Let $a=\operatorname{cf}(a)$. Then $\left(a^{+}, a\right) \rightarrow$ wk $\Delta\left(a^{+}\right)$.

Proof. By [3], $a^{+} \rightarrow\left(a^{+}\right)_{2}^{2}$. Hence there is a function $f:\left[a^{+}\right]^{2} \nrightarrow \underline{2}$ such that, whenever $M \subset \underline{a}^{+}$and $f$ is constant on $[M]^{2}$, then $|M|<a^{+}$. By Lemma 6, there are sets $F_{\gamma}$ such that $\left|F_{\gamma}\right|=a$ for $\gamma \in \underline{a}^{+}$and, for $\mu<\gamma \in \underline{a}^{+},\left|F_{\mu} \cap F_{\gamma}\right|<a$ if $f(\mu, \gamma)=0 ;\left|F_{\mu} \cap F_{\gamma}\right|=a$ if $f(\mu, \gamma)=1$. Then the $\left(a^{+}, a\right)$-system $\left(F_{\gamma}: \gamma \in \underline{a}^{+}\right)$has no wk $\Delta\left(a^{+}\right)$-subsystem.

Lemma 8. Let $a \rightarrow(c)_{\psi(b)}^{2}$. Then $(a, b) \rightarrow \mathrm{wk} \Delta(c)$.

Proof. Let $\left(A_{\gamma}: \gamma \in N\right) \in \Omega(a, b)$. Then

$$
[N]^{2}=\bigcup\left(b_{0} \leqq b\right)\left\{\{\mu, \gamma\}_{\#} \subset N:\left|A_{\mu} \cap A_{\nu}\right|=b_{0}\right\} .
$$

By Hypothesis there are $M$ and $b_{0}$ such that $M \in[N]^{c} ; b_{0} \leqq b ;\left|A_{\mu} \cap A_{\gamma}\right|=b_{0}$ for $\{\mu, \gamma\}_{\neq} \subset M$. Then

$$
\left(A_{\gamma}: \gamma \in M\right)_{h_{0}} \in \mathrm{wk} \Delta(c) \text {. }
$$

LEMMA 9. Let $a>a^{-}$. Then $\left(a^{+}, a\right) \rightarrow \mathrm{wk} \Delta(a)$.

PRoOF. $\psi(a)=\psi\left(a^{-}\right) \leqq a^{-}<a$. Hence, clearly, $a \rightarrow(a)_{\psi(a)}^{1}$ and therefore, by the "stepping-up lemma" of [3], $a^{+} \rightarrow(a)_{\psi(a)}^{2}$. Now Lemma 8 yields $\left(a^{+}, a\right) \rightarrow$ wk $\Delta(a)$.

Lemma 10. Let $(a, b) \rightarrow \mathrm{wk} \Delta(c)$. Then $\left(a^{\prime}, b^{\prime}\right) \rightarrow \mathrm{wk} \Delta\left(c^{\prime}\right)$ if $a \geqq a^{\prime} ; b \leqq b^{\prime}$; $c \leqq c^{\prime}$.

Remark. This lemma will be applied without reference.

Proof. There is an $(a, b)$-system $\left(A_{\gamma}: \gamma \in N\right)$ without wk $\Delta(c)$-subsystem. Choose sets $B_{\gamma}$ such that $A_{\gamma} \subset B_{\gamma}$ and $\left|B_{\gamma}\right|=b^{\prime}$ for $\gamma \in N$, and $\left(\left(B_{\gamma}-A_{\gamma}\right)_{\gamma \in N}\right.$, $\left.A_{N}\right)_{0}$. Let $N^{\prime} \in[N]^{a^{\prime}}$.Then the $\left(a^{\prime}, b^{\prime}\right)$-system $\left(B_{\gamma}: y \in N^{\prime}\right)$ has no wk $\Delta\left(c^{\prime}\right)$-subsystem.

LeMma $11 .(\psi(b), b) \rightarrow \mathrm{wk} \Delta(3)$.

Proof. Put $N=\underline{\omega} \cup\left\{\omega_{0}, \cdots, \hat{\omega}_{\beta}\right\}$

$$
A_{\gamma}=\underline{\gamma} \cup\left\{\xi: \omega_{\beta} \gamma \leqq \xi<\omega_{\beta}(\gamma+1)\right\}(\gamma \in N) .
$$


Then the $(\psi(b), b)$-system $\left(A_{\gamma} ; \gamma \in N\right)$ has no wk $\Delta(3)$-subsystem. For if $\{\mu, \gamma, \lambda\}_{<} \subset N$ then

$$
\left|A_{\mu} \cap A_{\gamma}\right|=|\mu|<|\gamma|=\left|A_{\gamma} \cap A_{\lambda}\right|
$$

LEMMA 12. Let $b=b^{-}$. Then $\left(b^{+}, b\right) \rightarrow \mathrm{wk} \Delta(b)$.

Proof. Put $N=\left\{\gamma=\left(\gamma_{0}, \cdots, \hat{\gamma}_{\omega_{\beta}}\right): \gamma_{0}, \cdots, \hat{\gamma}_{\omega_{\beta}} \in \underline{2}\right\}$

$$
A_{\gamma}=\left\{\left(\gamma_{0}, \cdots, \gamma_{\lambda}\right): \lambda \in \underline{b}\right\}(\gamma \in N) \text {. }
$$

Then $\left(A_{\gamma}: \gamma \in N\right) \in \Omega\left(b^{+}, b\right)$. Assume that there is $M \in[N]^{b}$ such that $\left(A_{\gamma}: \gamma \in M\right)_{p}$ for some $p$. Let $\{\mu, \gamma\}_{\neq} \subset M$. Then $p=\left|A_{\mu} \cap A_{\gamma}\right|=|\mu \circ \gamma|<b ; \mu \circ \gamma \in p^{+}$. Put $\sigma=\omega\left(p^{+}\right)$. Then $\mid\left\{\left(\gamma_{0}, \cdots, \hat{\gamma}_{\sigma}\right):\left(\gamma_{0}, \cdots, \hat{\gamma}_{\omega_{\beta}}\right) \in M\right.$ for some $\left.\gamma_{\sigma}, \cdots, \hat{\gamma}_{\omega_{\beta}}\right\} \mid \leqq 2^{|\sigma|}$ $=p^{++}<b=|M|$, and there is $\{\mu, \gamma\}_{\neq} \subset M$ such that $\left(\mu_{0}, \cdots, \hat{\mu}_{\sigma}\right)=\left(\gamma_{0}, \cdots, \hat{\gamma}_{\sigma}\right)$. On the other hand, if $\lambda=\mu \circ \gamma$ then $\lambda<\sigma ; \mu_{\bar{\lambda}} \neq \gamma_{\bar{\lambda}}$, which is a contradiction.

LEMMA 13. Let $b=\psi(b)$. Then $\left(b^{+}, b\right) \rightarrow \mathrm{wk} \Delta(3)$.

Proof. Case 1. $\beta=0$. The conclusion follows from the case $a=2 ; n=\aleph_{0}$ of Theorem 4 .

CASE 2. $\beta>0$. For $\lambda<\beta$ and $\gamma_{0}, \cdots, \hat{\gamma}_{\lambda} \in \underline{2}$, choose a set $X\left(\gamma_{0}, \cdots, \hat{\gamma}_{\lambda}\right)$ with $\left|X\left(\gamma_{0}, \cdots, \hat{\gamma}_{\lambda}\right)\right|=\aleph_{\lambda+1}$, such that $\left(X\left(\gamma_{0}, \cdots, \hat{\gamma}_{\lambda}\right): \lambda<\beta ; \gamma_{0}, \cdots, \hat{\gamma}_{\lambda} \in 2\right)_{0}$. Put $A_{\gamma}$ $=\bigcup(\lambda<\beta) X\left(\gamma_{0}, \cdots, \hat{\gamma}_{\lambda}\right)$ for $\gamma=\left(\gamma_{0}, \cdots, \hat{\gamma}_{\beta}\right) ; \gamma_{0}, \cdots, \hat{\gamma}_{\beta} \in \underline{2}$. Then $\left|A_{\gamma}\right|=$ $\Sigma(\lambda<\beta) \aleph_{\lambda+1}=\aleph_{\beta}=b$. We have $\left.\mid\left\{\gamma_{0}, \cdots, \hat{\gamma}_{\beta}\right): \gamma_{0}, \cdots, \hat{\gamma}_{\beta} \in \underline{2}\right\}\left.\left|=2^{|\beta|}=\right| \beta\right|^{+}$ $=b^{+}$. Let $(\mu, \gamma, \rho)_{\neq}$and $\left(A_{\mu}, A_{\gamma}, A_{\rho}\right) \in \mathrm{wk} \Delta(3)$. Put $\mu \circ \gamma=\tau$.

We note that $\left\{\lambda:\left(\mu_{0}, \cdots, \hat{\mu}_{\lambda}\right)=\left(\gamma_{0}, \cdots, \hat{\gamma}_{\lambda}\right)\right\}=\underline{\tau+1}$. Hence $\left|A_{\mu} \cap A_{\gamma}\right|$ $=\left|\bigcup(\lambda<\tau+1) X\left(\gamma_{0}, \cdots, \hat{\gamma}_{\lambda}\right)\right|=\Sigma(\lambda<\tau+1) \aleph_{\lambda+1}=\bar{\aleph}_{\tau+1}=\aleph_{\mu \circ \gamma+1}$. Therefore $\tau=\mu \circ \gamma=\mu \circ \rho=\gamma \circ \rho$, and $\left(\mu_{\tau}, \gamma_{\tau}, \rho_{\tau}\right)_{\neq}$which is impossible. This proves Lemma 13.

LemMA 14. Let $\operatorname{cf}(d)=\aleph_{0}$. Then $\left(d^{+}, \aleph_{0}\right) \rightarrow$ wk $\Delta(d)$.

Proof. There are cardinals $d_{\lambda}$ such that $d_{0}, \cdots, \hat{d}_{\omega}<d=d_{0}+\cdots+\hat{d}_{\omega}$. Put

$$
X=\left\{x=\left(x_{0}, \cdots, \hat{x}_{\omega}\right): x_{\lambda} \in \underline{d}_{\lambda}(\lambda<\omega)\right\} ;
$$

$A_{x}=\left\{\left(x_{0}, \cdots, \hat{x}_{\lambda}\right): \lambda<\omega\right\}(x \in X)$. Then $\left(A_{x}: x \in X\right) \in \Omega\left(d^{+}, \aleph_{0}\right)$. Let $L \subset X$ and $\left(A_{x}: x \in L\right) \in$ wk $\Delta$. Then there is $\sigma<\omega$ such that $\left|A_{x} \cap A_{y}\right|=\sigma+1 ; x \circ y=\sigma$ for $\{x, y\}_{\neq} \subset L$. Then $|L|=\left|\left\{x_{\sigma}: x \in L\right\}\right| \leqq d_{\sigma}<d$ which proves Lemma 14.

Lemma 15. Let $\operatorname{cf}(d)=\aleph_{\delta}$. Then $\left(d^{+}, \aleph_{\omega_{\delta}}\right) \rightarrow$ wk $\Delta(d)$.

PROOF. There are cardinals $d_{\lambda}$ such that $d_{0}, \cdots, \hat{d}_{\omega_{\delta}}<d=d_{0}+\cdots+\hat{d}_{\omega_{\delta}}$. Let 
$X=\left\{x=\left(x_{0}, \cdots, \hat{x}_{\omega_{\delta}}\right): x_{y} \in \underline{d}_{\gamma}\left(\gamma<\omega_{\delta}\right)\right\}$. For $x \in X$ and $\lambda<\omega_{\delta}$, let $\mid B\left(x_{0}\right.$, $\left.\cdots, \hat{x}_{\lambda}\right) \mid=\aleph_{\lambda+1}$, and $\left(B\left(x_{0}, \cdots, \hat{x}_{\lambda}\right): \lambda<\omega_{\delta} ; x_{\gamma} \in \underline{d}_{\gamma}(\gamma<\lambda)\right)_{0}$. Put

$$
A_{x}=\bigcup\left(\lambda<\omega_{\hat{\kappa}}\right) B\left(x_{0}, \cdots, \hat{x}_{\lambda}\right)
$$

for $x \in X$. Then $|X|=d_{0} \cdots \hat{d}_{\omega_{\delta}}=d^{+} ;\left|A_{x}\right|=\Sigma\left(\lambda<\omega_{\delta}\right) \aleph_{\lambda+1}=\aleph_{\omega_{\delta}}$, so that $\left(A_{x}: x \in X\right) \in \Omega\left(d^{+}, \aleph_{\omega_{\delta}}\right)$. Let $L \subset X$ and $\left(A_{x}: x \in L\right) \in \mathrm{wk} \Delta$. Then there is $\sigma<\omega_{\delta}$ such that $x \circ y=\sigma$ for $\{x, y\}_{\neq} \subset L$. Hence $|L|=\left|\left\{x_{\sigma}: \sigma \in L\right\}\right| \leqq d_{\sigma}<d$, which completes the proof.

Lemma 16. Let $0<d=d^{-}<\aleph_{\omega_{n}}$. Then $\operatorname{cf}(d)<\aleph_{n}$.

ProOF. We have $d=\aleph_{\delta}$ for some $\delta<\omega_{n}$. Since $d=d^{-}$we conclude that $d=\Sigma(\pi<\delta) \aleph_{\pi} ; \operatorname{cf}(d) \leqq|\delta|<\aleph_{n}$.

For the last two lemmas we need the following definitions: Consider a system $\mathscr{F}=\left(A_{\gamma}: \gamma \in N\right)$. We call $\mathscr{F}$ an $(a, b, \leqq d)$-system if $\mathscr{F} \in \Omega(a, b)$ and $\left(A_{\gamma}\right.$ : $\gamma \in N)_{\leqq d}$. An $(a, b,<d)$-system is defined similarly. For every set $A$ and every cardinal $d$ we put

$$
\mathscr{F}(A, d)=\left\{\gamma \in N:\left|A \cap A_{\gamma}\right|=d\right\} .
$$

Lemma 17. Let $\mathscr{F}$ be an $(a, b, \leqq d)$-system; $a=\operatorname{cf}(a)>b^{d} ;|A|=b$; $|\mathscr{F}(A, d)|=a$. Then $\mathscr{F}$ has a wk $\Delta(a)$-subsystem.

Proof. We have $\left|[A]^{d}\right|=b^{d}<a=\operatorname{cf}(a)$. Hence there is an $(a, b)$-subsystem $\mathscr{F}^{\prime}=\left(A_{\gamma}: \gamma \in N^{\prime}\right)$ of $\mathscr{F}$ and a set $X$ such that $|X|=d$ and $A \cap A_{\gamma}=X\left(\gamma \in N^{\prime}\right)$. Then, for $\{\mu, \gamma\}_{\neq} \subset N^{\prime}$, we have $d=|X| \leqq\left|A_{\mu} \cap A_{\gamma}\right| \leqq d$, and $\mathscr{F}^{\prime}$ is a wk $\Delta(a)$ system.

LEMMA 18. Let $\mathscr{F}=\left(A_{\gamma}: \gamma \in N\right)$ be an $(a, b, \leqq d)$-system, such that

$$
\left|\mathscr{F}\left(A_{\gamma}, d\right)\right|<a
$$

for every $\gamma \in N$. Suppose that $a=\operatorname{cf}(a)$. Then $\mathscr{F}$ has an $(a, b,<d)$-subsystem.

Proof. Assume $N=\underline{a}$. We can construct inductively ordinals $\gamma_{\rho}$ for $\rho \in \underline{a}$ such that, for each $\rho \in \underline{a}, \gamma_{\rho} \in\left(N-\bigcup(\sigma<\rho) \mathscr{F}\left(A_{\gamma_{-}}, d\right)\right)-\left\{\gamma_{0}, \cdots, \hat{\gamma}_{\rho}\right\}$. Then $\left(A_{\gamma_{\rho}}: \rho \in \underline{a}\right)$ is an $(a, b,<d)$-system.

\section{Discussion of the $w k \Delta$-relation}

We consider two fixed infinite cardinals $a, b$, where

$$
a=\aleph_{\alpha} ; b=\aleph_{\beta},
$$


and we shall determine all cardinals $c$ such that the wk $\Delta$-relation

$$
\text { - }(a, b) \rightarrow \text { wk } \Delta(c)
$$

is true. There is a least cardinal $\phi(a, b)$ in $3 \leqq \phi(a, b) \leqq a^{+}$such that (7) holds if and only if $c<\phi(a, b)$. We shall determine $\phi(a, b)$. If $\phi(a, b)=3$ then (7) only holds completely trivially, i.e. for $c \leqq 2$, whereas $\phi(a, b)=a^{+}$means that (7) holds for all values of $c$ which are at all admissible, which are the cardinals $c \leqq a$.

Our results show that, for all $a, b$.

$$
\phi(a, b) \in\left\{3, a^{-}, a, a^{+}\right\} .
$$

In our discussion we shall write $\phi$ instead of $\phi(a, b)$. We remind the reader that throughout this section we assume GCH.

CASE 1. $a>b^{+}$.

CASE 1a. $a>a^{-}>a^{--}$. We prove that $\phi=a^{+}$. We can write $a=a_{0}^{++}$, and then we have $a_{0}^{++}=a \geqq b^{++} ; a_{0} \geqq b$. By [2], Theorem 1 (ii), with $a, b$ in [2] replaced by $a_{0}^{+}, a_{0}$ respectively, we have $\left(a_{0}^{++}, a_{0}\right) \rightarrow$ st $\Delta\left(a_{0}^{++}\right)$. Hence $(a, b) \rightarrow$ st $\Delta(a)$.

CASE 1b. $a>a^{-}=a^{--}$.

CASE 1b1. $b<\operatorname{cf}\left(a^{-}\right)$. Then $\phi=a^{+}$. Indeed, by Lemma $4,(a, b) \rightarrow$ st $\Delta(a)$.

CASE 1b2. $b \geqq \operatorname{cf}\left(a^{-}\right)$. Let $a_{0}<a^{-}$. Put $a_{1}=\max \left\{a_{0}, b\right\}$. Then $\left(a_{1}^{++}, a_{1}\right)$ $\rightarrow$ st $\Delta\left(a_{1}^{++}\right)$by [2]. Hence $(a, b) \rightarrow$ st $\Delta\left(a_{0}\right)\left(a_{0}<a^{-}\right)$.

CASE 1b2a. $\operatorname{cf}\left(a^{-}\right)=\operatorname{cf}^{-}\left(a^{-}\right)$.

CASE 1b2a1. $\operatorname{cf}\left(a^{-}\right)=\aleph_{0}$. Then $\phi=a^{-}$. For, by Lemma 14, $\left(a, \aleph_{0}\right)$ $\rightarrow \mathrm{wk} \Delta\left(a^{-}\right)$and therefore $(a, b) \rightarrow \mathrm{wk} \Delta\left(a^{-}\right)$.

CASE 1b2a2. $\operatorname{cf}\left(a^{-}\right)>\aleph_{0}$. Then $\phi=a^{-}$. For, we have, by Lemma 15 , $\left(a, \operatorname{cf}\left(a^{-}\right)\right)+\mathrm{wk} \Delta\left(a^{-}\right)$.

To see this, put $\operatorname{cf}\left(a^{-}\right)=\aleph_{\delta}$. Then $\delta$ is a positive limit ordinal; $\aleph_{\delta}=\operatorname{cf}\left(\aleph_{\delta}\right)$. If $\delta<\omega_{0}$ then $\aleph_{\delta}=\Sigma\left(\delta_{0}<\delta\right) \aleph_{\delta_{n}} ; \operatorname{cf}\left(\aleph_{\delta}\right) \leqq|\delta|<\aleph_{\delta}$, which is false. Hence $\delta=\omega_{\delta}$. By Lemma 15 , with $d=a^{-}$, we have $\left(a, \aleph_{\omega_{A}}\right) \rightarrow$ wk $\Delta\left(a^{-}\right)$, i.e. $\left(a, \operatorname{cf}\left(a^{-}\right)\right)$ $\rightarrow \mathrm{wk} \Delta\left(a^{-}\right)$. This implies $(a, b) \rightarrow \mathrm{wk} \Delta\left(a^{-}\right)$.

CASE 1b2b. $\operatorname{cf}\left(a^{-}\right)>\operatorname{cf}^{-}\left(a^{-}\right)$. Then $\operatorname{cf}\left(a^{-}\right)$has the form $\aleph_{\lambda+1}$.

CASE 1b2b1. $\aleph_{\omega_{\lambda+1}} \leqq b$. Then $\phi=a^{-}$. For, by Lemma $15,\left(a, \aleph_{\omega_{\lambda+1}}\right)$ $\rightarrow \mathrm{wk} \Delta\left(a^{-}\right)$, which implies $(a, b) \rightarrow \mathrm{wk} \Delta\left(a^{-}\right)$. 
CASE 1b2b2. $\aleph_{\omega_{\lambda+1}}>b$. We show that $\phi=a^{+}$. We use the notation $\mathscr{F}(A, d)$ introduced before the statement of Lemma 17. We assume that the $(a, b)$-system $\mathscr{F}$ has no wk $\Delta(a)$-subsystem, and we have to deduce a contradiction. Since $\mathscr{F}$ is an $(a, b, \leqq b)$-system, it follows that there is a least cardinal $d$ such that $\mathscr{F}$ has an $(a, b, \leqq d)$-subsystem. We have $0<d \leqq b$. We may assume that $\mathscr{F}$ itself is an $(a, b, \leqq d)$-system. Then $\mathscr{F}$ has no $(a, b, \leqq e)$-subsystem, for every $e<d$. Let $\mathscr{F}=\left(A_{\gamma}: \gamma \in N\right)_{\leqq d}$. Let $\gamma_{0} \in N$ and $\left|\mathscr{F}\left(A_{\gamma_{\gamma}}, d\right)\right|=a$. Since $b^{d} \leqq b^{b}=b^{+}<a$, it follows from Lemma 17 that $\mathscr{F}$ has a wk $\Delta(a)$-subsystem, which is a contradiction. Hence $\left|\mathscr{F}\left(A_{\gamma}, d\right)\right|<a$ for $\gamma \in N$. Then, by Lemma 18, $\mathscr{F}$ has an $(a, b,<d)$ subsystem. We may assume that $\mathscr{F}=\left(A_{\gamma}: \gamma \in N\right)_{<_{d}}$ is itself an $(a, b,<d)$-system. If $d=e^{+}$, then $\mathscr{F}$ is an $(a, b$, $\leqq e)$-system, which contradicts the minimality of $d$. Hence $0<d=d^{-} \leqq b<\aleph_{\omega_{\lambda+1}}$ and, by Lemma $16, \operatorname{cf}(d)<\aleph_{\lambda+1}$.

We shall now construct a modified $d$-sequence. There is a maximal set $N_{0} \subset N$ such that $\left(A_{\gamma}: \gamma \in N_{0}\right)_{0}$. Then $0<\left|N_{0}\right|<a$. Let $0<\sigma \in \underline{a}$. Suppose that, for each $\rho<\sigma$, we have already defined a set $N_{\rho} \in[N]^{<a}$, where $N_{\rho} \neq \varnothing$, such that, putting $S_{\rho}=A_{N_{\rho}}$, we have $\left|A_{\gamma} \cap S_{\rho}\right|<d$ for $\gamma \in N_{\rho} ; A_{\mu} \cap A_{\gamma} \subset S_{\rho}$ for $\{\mu, \gamma\}_{\neq} \subset N_{\rho}$. Suppose, furthermore, that, for each $\rho<\sigma$, the set $N_{\rho}$ is maximal such that the above stated conditions hold, i.e.: if $\gamma \in N-N_{\rho}$, then either $A_{\gamma} \subset S_{\rho}$, or there is $\mu \in N_{\rho}-\{\gamma\}$ with $A_{\mu} \cap A_{\gamma} \notin S_{\rho}$. We shall now define $N_{\sigma}$, and in such a way that all these conditions hold for $\rho=\sigma$. Put $S_{\sigma}=A_{N_{\sigma}}$. Then $\left|S_{\sigma}\right| \leqq|\sigma| a^{-} b \sigma$ $=a^{-}$. Well-order $S_{\sigma}$ by a relation $\prec$, so that $\operatorname{tp}\left(\bar{S}_{\sigma}, \prec\right) \leqq \omega\left(a^{-}\right)$. Put $N^{*}$ $=\left\{\gamma \in N:\left|A_{\gamma} \cap S_{\sigma}\right| \geqq d\right\}$. We now prove $\left|N^{*}\right|<a$. Assume $\left|N^{*}\right|=a$. For each $\gamma \in N^{*}$, denote by $g(\gamma)$ the initial section of $\left(A_{\gamma} \cap S_{\sigma}, \precsim\right)$ of type $\omega(d)$. If $\{\mu, \gamma\}_{\neq} \subset N^{*}$ then, by $\left(A_{\gamma}: \gamma \in N\right)_{<d}$, we have $\left|A_{\mu} \cap A_{\gamma}\right|<d$, and hence $g(\mu)$ $\neq g(\gamma)$. There is an initial section $T$ of $\left(S_{\sigma}, \prec\right)$ such that $|T|<a^{-}$and $\mid\left\{\gamma \in N^{*}\right.$ : $g(\gamma) \subset T\} \mid=a$. For: if $\left|S_{\sigma}\right|<a^{-}$then we put $T=S_{\sigma}$. Now let $\left|S_{\sigma}\right|=a^{-}$. We have $\operatorname{cf}(d)<\aleph_{\lambda+1}=\operatorname{cf}\left(a^{-}\right)$. For each $\gamma \in N^{*}$, the set $(g(\gamma), \prec)$ has a cofinal subset of cardinal $\operatorname{cf}(d)$. This subset is not cofinal in $\left(S_{\sigma}, \zeta\right)$. Hence $g(\gamma)$ is not cofinal in $\left(S_{\sigma}, \prec\right)$, and there is $x_{y} \in S_{\sigma}$ such that $g(\gamma) \subset\left\{x \in S_{\sigma}: x \prec x_{y}\right\}$. In view of $a=\operatorname{cf}(a)$, there is $x^{*} \in S_{\sigma}$ such that $\left|\left\{\gamma \in N^{*}: x_{\gamma}=x^{*}\right\}\right|=a$. Then we may put $T=\left\{x \in S_{\sigma}: x \prec x^{*}\right\}$. This completes the definition of $T$. Now we have $\left|[T]^{d}\right| \leqq 2^{|T|} \leqq a^{-}$. Hence there is $X \subset T$ such that $\left|\left\{\gamma \in N^{*}: g(\gamma)=X\right\}\right|=a$. But then $\left(A_{\gamma}: \gamma \in N^{*} ; g(\gamma)=X\right)_{\geq d}$, which contradicts the relation $\left(A_{\gamma}: \gamma \in N\right)_{<d}$.

We have thus proved $\left|N^{*}\right|<a$. Let $\gamma \in N-N^{*}$. If $A_{\gamma} \subset S_{\sigma}$ then we have $b=\left|A_{\gamma}\right|=\left|A_{\gamma} \cap S_{\sigma}\right|<d \leqq b$ which is false. Hence $\gamma \in N-N^{*}$ implies $A_{\gamma} \notin S_{\sigma}$. Let $N_{\sigma}$ be maximal such that $N_{\sigma} \subset N-N^{*}$ and $\left(A_{y}-S_{\sigma}: \gamma \in N_{\sigma}\right)_{0}$. Then $N_{\sigma} \neq \varnothing$ It follows that if $\gamma \in N_{\sigma}$ then $A_{\gamma} \notin S_{\sigma}$, and if $\{\mu, \gamma\}_{\neq} \subset N_{\sigma}$ then $A_{\mu} \cap A_{\gamma} \subset S_{\sigma}$. Also, if $\gamma \in N-N_{\sigma}$ and $\left|A_{\gamma} \cap S_{p}\right|<d$, then there is $\mu \in N_{\sigma}$ with $A_{\mu} \cap A_{\gamma} \notin S_{\sigma}$. In order to complete the inductive definition of $N_{0}, N_{1}, \cdots$ we must now show that $\left|N_{\sigma}\right|<a$. Assume that $\left|N_{\sigma}\right|=a$. Corresponding to every $\gamma \in N_{\sigma}$, there is $e_{\gamma}<d$ such that $\left|A_{\gamma} \cap S_{\sigma}\right|=e_{\gamma}$. Then there is $e<d$ such that $\left|\left\{\gamma \in N_{\sigma}: e_{\gamma}=e\right\}\right|=a$. For we 
have $\left|\left\{e_{\gamma}: \gamma \in N_{\sigma}\right\}\right| \leqq d \leqq b<a^{-}$. Put $N^{\prime}=\left\{\gamma \in N_{\sigma}:\left|A_{\gamma} \cap S_{\sigma}\right|=e\right\}$, so that $\left|N^{\prime}\right|=a$. If $\{\mu, \gamma\}_{\neq} \subset N^{\prime}$, then $\left|A_{\mu} \cap A_{\gamma}\right|=\left|A_{\mu} \cap A_{\gamma} \cap S_{\sigma}\right| \leqq\left|A_{\mu} \cap S_{\sigma}\right|=e$. Hence $\left(A_{\gamma}: \gamma \in N^{\prime}\right)_{\leqq} \in \Omega(a, b)$ which contradicts the minimum property of $d$. This proves $\left|N_{\sigma}\right|<a$, and the inductive definition of $N_{\rho}$ for $\rho \in a$ is accomplished. We have $b^{+}<a$, and therefore we can choose $\gamma \in N_{\underline{\omega}\left(b_{+}\right)}$. For each $\rho \in \underline{b}^{+}$there is $\mu_{\rho} \in N_{\rho}$ such that $A_{\mu_{\rho}} \cap A_{\gamma} \notin S_{\rho}=A_{N_{\rho}}$. We can choose $z_{\rho} \in A_{\mu} \cap A A_{\gamma}-A_{N_{\rho}}$. If $\tau<\rho$ then $z_{\tau} \in A_{\mu_{-}} \cap A_{\gamma} \subset A_{\mu_{\tau}} \subset A_{N_{\rho}}$. Hence $z_{\rho} \neq z_{\tau}$ for $\tau<\rho \in \underline{b}^{+}$;

$$
\left|A_{\gamma}\right| \geqq\left|\left\{z_{\rho}: \rho \in \underline{b}^{+}\right\}_{\neq}\right|=b^{+}>b=\left|A_{\gamma}\right| \text {. }
$$

which is the required contradiction.

CASE 1c. $a=a^{-}$.

CASE 1c1. $a=c f(a)$. Then $\phi=a^{+}$. For, by Lemma $5,(a, b) \rightarrow s t \Delta(a)$.

CASE 1c2. $a>\operatorname{cf}(a)$. Then $\phi=a$. For, by Lemma $3,(a, b) \rightarrow \mathrm{wk} \Delta(a)$. Let $a_{0}<a$ and put $a_{1}=\max \left\{a_{0}, b\right\}$. Then, by [2], $\left(a_{1}^{++}, a_{1}\right) \rightarrow$ st $\Delta\left(a_{1}^{++}\right)$. Hence $(a, b) \rightarrow \operatorname{st} \Delta\left(a_{0}\right)\left(a_{0}<a\right)$.

CASE 2. $a=b^{+}$.

CASE 2a. $b=|\beta|$. Then $\phi=3$. For, by Theorem $5,\left(2^{|\beta|}, b\right) \rightarrow \mathrm{wk} \Delta(3)$. Hence $(a, b) \rightarrow \mathrm{wk} \Delta(3)$.

CASE 2b. $b>|\beta|$.

CASE 2b1. $b>b^{-}$. Then $\phi=a$. For, by Lemma $7,(a, b) \rightarrow \mathrm{wk} \Delta(a)$. Also, by Lemma $9,(a, b) \rightarrow$ wk $\Delta(b)$.

CASE 2b2. $b=b^{-}$. Then $\phi=a^{-}$. For, by Lemma $12,(a, b) \rightarrow \mathrm{wk} \Delta(b)$. Now, let $b_{0}<b$. Then, by [3], $b \rightarrow\left(b_{0}\right)_{\psi(b)}^{2}$, and Lemma 8 gives $(b, b) \rightarrow \operatorname{wk} \Delta\left(b_{0}\right)$. Hence $(a, b) \rightarrow$ wk $\Delta\left(b_{0}\right)\left(b_{0}<b\right)$.

CASE 3. $a=b$.

CASE 3a. $b=|\beta|$. Then $\phi=3$. For, by Lemma $11,(a, b) \rightarrow$ wk $\Delta(3)$.

CASE 3b. $b>|\beta|$.

CASE $3 \mathrm{~b} 1 . b>b^{-}$. If $b^{-}=\operatorname{cf}\left(b^{-}\right)$then, by Lemma $7,\left(b, b^{-}\right)+\mathrm{wk} \Delta(b)$, and if $b^{-}>\operatorname{cf}\left(b^{-}\right)$then, by Lemma $12,\left(b, b^{-}\right) \rightarrow \mathrm{wk} \Delta\left(b^{-}\right)$. Thus, in either case, $(a, b) \rightarrow \mathrm{wk} \Delta(b)$.

CASE 3b1a. $b^{-}>b^{--}$. Then $\phi=a$. For we have $\beta=\beta_{0}+1=\beta_{1}+2$ for some $\beta_{0}, \beta_{1} ; \psi(b)=\aleph_{0}+\left|\beta_{1}\right| ; \aleph_{\beta_{1}+1} \rightarrow\left(\aleph_{\beta_{1}+1}\right)_{\psi(b)}^{1}$ and, by [3], $\aleph_{\beta_{1}+2} \rightarrow\left(\aleph_{\beta_{1}+1}\right)_{\psi(b)}^{2}$. Now Lemma 8 gives $(a, b) \rightarrow$ wk $\Delta\left(b^{-}\right)$.

CASE 3b1b. $b^{-}=b^{--}$. Then, by Lemma $12,\left(b, b^{-}\right) \rightarrow \mathrm{wk} \Delta\left(b^{-}\right)$and hence $(a, b) \rightarrow \mathrm{wk} \Delta\left(b^{-}\right)$. 
CASE 3b1b1. $\psi\left(b^{-}\right)=b^{-}$. Then $\phi=3$. For, by Lemma $13,\left(b, b^{-}\right) \rightarrow$ wk $\Delta(3)$. Hence $(a, b) \rightarrow \mathrm{wk} \Delta(3)$.

CASE 3b1b2. $\psi\left(b^{-}\right)<b^{-}$. Then $\phi=a^{-}$. For, let $b_{0}<b^{-}$. Then $b \rightarrow$ $\left(b_{0}\right)_{\psi(b)}^{2}$ and, by Lemma 8 ,

$$
(a, b) \rightarrow \mathrm{wk} \Delta\left(b_{0}\right)\left(b_{0}<b^{-}\right) .
$$

CASE 3b2. $b=b^{-}$. Then $\phi=a$. For, by Lemma $12,\left(b^{+}, b\right) \rightarrow \mathrm{wk} \Delta(b)$, and hence $(a, b)+\mathrm{wk} \Delta(b)$. Let $b_{0}<b$. Then $b \rightarrow\left(b_{0}\right)_{\psi(b)}^{2}$ and, by Lemma 8 ,

$$
(a, b) \rightarrow \mathrm{wk} \Delta\left(b_{0}\right)\left(b_{0}<b\right) \text {. }
$$

CASE 4. $a<b$.

CASE 4a. $b=|\beta|$. Then $\phi=3$. For, by Lemma $11,(\psi(b), b) \rightarrow \mathrm{wk} \Delta(3)$ and hence $(a, b) \nrightarrow \mathrm{wk} \Delta(3)$.

CASE $4 \mathrm{~b} . b>|\beta|$.

CASE $4 \mathrm{~b} 1 . a \leqq 2^{|\beta|}$. Then $\phi=3$. For, by Theorem $5,\left(2^{|\beta|}, b\right) \rightarrow \mathrm{wk} \Delta(3)$ and therefore $(a, b) \rightarrow \mathrm{wk} \Delta(3)$.

CASE 4b2. $a>2^{\aleph_{0}+|\beta|}$. Then $|\beta|<2^{|\beta|}<a$.

CASE 4b2a. $a=a^{-}$. Then $\phi=a$. For, by Lemma 12, $\left(a^{+}, a\right) \rightarrow \mathrm{wk} \Delta(a)$, and therefore $(a, b) \rightarrow \mathrm{wk} \Delta(a)$. Let $a_{0}<a$. Then $a \rightarrow\left(a_{0}\right)_{\mathrm{N}_{0}+|\beta|}^{2}$, and Lemma 8 gives $(a, b) \rightarrow$ wk $\Delta\left(a_{0}\right)\left(a_{0}<a\right)$.

CASE $4 \mathrm{~b} 2 \mathrm{~b} . a>a^{-}$.

CASE 4b2b1. $a^{-}>a^{--}$. Then $\phi=a$. For: $|\beta|<2^{|\beta|}<a ; a^{-} \rightarrow\left(a^{-}\right)_{\aleph_{0}+|\beta|}^{1} ;$ $a \rightarrow\left(a^{-}\right)_{\psi(b)}^{2} ;(a, b) \rightarrow \operatorname{wk} \Delta\left(a^{-}\right)$. By Lemma $7,\left(a, a^{-}\right) \rightarrow$ wk $\Delta(a)$. Since $a^{-}<a$ $<b$, we deduce $(a, b) \rightarrow$ wk $\Delta(a)$.

CASE 4b2b2. $a^{-}=a^{--}$. Then $\phi=a^{-}$. For, Lemma 12 yields $\left(a, a^{-}\right) \rightarrow$ wk $\Delta\left(a^{-}\right)$, and hence $(a, b) \rightarrow \mathrm{wk} \Delta\left(a^{-}\right)$. Let $a_{0}<a^{-}$. Then $a^{-} \rightarrow\left(a_{0}\right)_{\mathbb{N}_{0}+|\beta|}^{1}$; $a \rightarrow\left(a_{0}\right)_{\psi(b)}^{2} ;(a, b) \rightarrow \mathrm{wk} \Delta\left(a_{0}\right)\left(a_{0}<a^{-}\right)$.

CASE 4b3. $2^{|\beta|}<a \leqq 2^{\aleph_{0}+|\beta|}$. Then $\phi=3$. For, we have $\beta<\omega$ and $a \leqq \aleph_{1}$. By Lemma 13, $\left(\aleph_{1}, \aleph_{0}\right) \rightarrow \mathrm{wk} \Delta(3)$. Hence $(a, b) \rightarrow \mathrm{wk} \Delta(3)$.

This concludes the dicsussion of the relation $(a, b) \rightarrow$ wk $\Delta(c)$ for infinite cardinals $a, b$.

\section{Acknowledgement}

The authors would like to thank the referee for his most helpful suggestions and criticism, and in particular for having supplied the proof of Lemma 6, which is simpler than the original proof. 


\section{References}

[1] P. Erdös and R. Rado, 'Intersection theorems for systems of sets', J. London Math. Soc. 35 (1960), 85-90.

[2] P. Erdös and R. Rado, 'Intersection theorems for systems of sets (II)', J. London Math. Soc. 44 (1969), 467-479.

[3] P. Erdös, A. Hajnal and R. Rado, 'Partition relations for cardinal numbers', Acta Math. Acad. Scient. Hungarica 16 (1965), 93-196.

University of Reading

England

University of Calgary

Canada 